\title{
An analysis of Interactive experience based on the gesture recognition VR system
}

\author{
XueqiaoFan ${ }^{1, a}$,Lei Zhu ${ }^{2, b}$ and HongZhang ${ }^{3, c}$ \\ ${ }^{1}$ Harbin Institute of Technology, China \\ ${ }^{2}$ Harbin Institute of Technology, China \\ ${ }^{3}$ Harbin Institute of Technology, China \\ afan_xueqiao@qq.com, 'leizcn@163.com, ̌hangh@hit.edu.cn
}

Keywords:VR system, gestural recognition, Performance measurement, emotional evaluation of consumers.

Abstract.With the development of the headset VR equipment of consumer-level and the interactive technology of the headset VR, especially the non-contacting gestural recognition technology in machine vision. The headset VR equipment of gestural recognition became the potential research area in the close interaction. This paper major researches the performance measurement of the VR system of gestural recognition in the Interactive experience the combination between subjective and objective methods. Lastly, the paper provides reference for gestural interaction design based on machine vision by the results of the accuracy of gestural recognition and the emotional evaluation of consumers.

\section{Methodology}

\section{Performance measurement}

Performance measurement is a technical term in the management which is a basic department of the incentives0, and means a measurement of administrative and innovation ability for the organization or unit to reflect the organization's ability for production, innovation, and other comprehensive level0. A system that can be used to evaluate, measure and evaluate the performance of the objects which be evaluated by the internal connections. The system normally uses a certain mathematical method to quantitatively or qualitatively evaluate the collected indexes. For the indexes of performance measurement, the selection standard major focuses on the information, sensitivity, accuracy, and consistency. The Information represents the level of selected indexes reflecting the research objects. The sensitivity means the strength and speed of the reactions. The accuracy refers the reliability of the selected indexes in the measurement. The consistency states the relationship between performance measurement and production. If the performance measurement equals production, the consistency is correct.

There are fore situations about performance measurement, which are Single-task single-indicator metric, single-task multi-index metric, multi-task single-index metric, and multitasking multi-index metric. Performance measurement argues that the ability of consumers used a VR production to achieve the aim in the evaluation of availability. Performance measurement relies on the setting of the tasks because of the overall measurement establish on the former designed task and exactly indexes of completion. The Performance measurement covers some parameters, such as completion of task, time limitation, mistake, and effectiveness. The completion of a task states that the ability of consumers effectively finishing a series tasks based on the dichotomy for quantification in general. The time limitation is about the finishing time of the task[3]. The measurement of mistakes used to find the production's problem, at the same time, combined with a think-aloud method to find the confusion from the consumers. The effectiveness measures the effort of finishing task and the amount of finishing task during the unit time.

$$
\text { Dichotomy successful }=\left\{\begin{array}{l}
1 \text { success } \\
0 \text { failure }
\end{array}\right.
$$




$$
\begin{gathered}
\bar{t}=\frac{1}{n} \sum_{i=1}^{n} t_{1}+t_{2}+\ldots+t_{n} \\
N=\sum_{i=1}^{n} n_{1}+n_{2}+\ldots+n_{n} \\
\eta_{i}=x_{i} / t_{i}(i=1,2, \ldots, n)
\end{gathered}
$$

$$
\begin{array}{cl}
\text { Among them } \bar{t}-\quad \begin{array}{l}
\text { Average time of the task } \\
N-
\end{array} \begin{array}{l}
\text { Number of subject } \\
n-
\end{array} \\
\text { Number of errors } \\
\eta_{i} \quad \text { Effectiveness }
\end{array}
$$

\section{Method of emotional evaluation}

According to ITU-T P910“ITU-T Recommendation P.910 Subjective video quality assessment methods for multimedia applications", ACR (Absolutely Category Rate)from the method of Single stimulation evaluation. The consumers use excellent, good, moderate, bad, and terrible to describe the emotion of the used procedure.In the ACR, when the tester finished a test, will have an independent mark. The VR equipment is evaluated by tester based on the evaluation standard.According to the method used in the subjective evaluation method of single stimulus, the time, correct rate and sensitivity in the gesture recognition process of VR equipment are scored using the scoring standard as shown in Table 1.

Table 1 ACR5 Experimental process

\begin{tabular}{|c|c|c|c|c|c|}
\hline Use device experience & excellent & good & moderate & bad & terrible \\
\hline score & 5 & 4 & 3 & 2 & 1 \\
\hline
\end{tabular}

\section{measurement process}

\section{Measurement equipment and gestural combinations.}

The Virtual reality display devices include virtual reality helmets, smart phones, data transmission lines, computers, and Bluetooth,as shown in figure 1. The Virtual reality helmet is a holder for the smartphone to show the virtual reality effects by the smartphone's screen though the two eyepieces. Moreover, the Virtual reality helmet which has an equipment of gestural recognition, transfers information with smartphone and computer by Bluetooth.

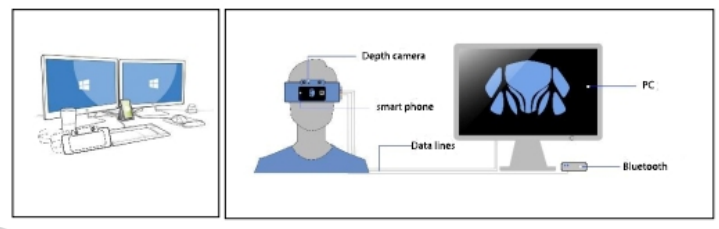

Fig.1Hardware equipment

The main experimental device:Inter ${ }^{\circledR}$ Real sense ,gesture recognizer, workbench, computer and bluetooth equipment

The equipment uses three - dimensional gestural recognition technology with two cameras recognition mode. According to the marketing research and product trial,Leap Motion is selected to be the gestural recognition equipment in the test considering price, size, useful, and identify system to test theidentification efficiency and identification area of the natural gestures recognized, as shown in figure2. 


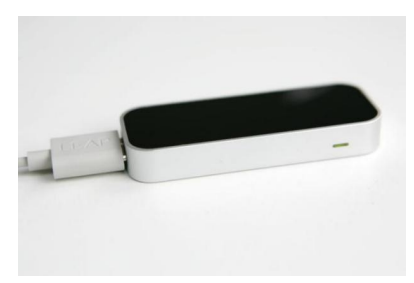

Fig.2 Leap Motion

The development environment of the gesture identification library:Operating system: Windows 10;Development platform: VS2010;SDK: Leap_Motion_SDK_Windows_3.1.3

The development of gesture recognition is based on the data collected by Leap Motion. Leap Motion can capture up to $120 \mathrm{fps}$. The data for each frame contains the spatial coordinates of 44 joints on each of the left and right hands, each of whose fingers include four joint space coordinates and the spatial coordinates of the wrist and palm. Gesture recognition is based on the coordinates of these joints.According to the position of the joint space coordinates on each frame,the gesture recognition is implemented.

Development Process:(1) to configure the related attributes of the project;(2) to obtain the data of each frame, and storage it; (3) to determine the relationship between the coordinates of each frame from the latest few pieces of data, to determine what kind of gestures people make in the current frame of data according to the characteristics of the hand gesture; (4) the algorithm will be packaged into a dynamic link library, that is, secondary development will be made, which is based on Leap Motion.The encapsulated dynamic link library can be re-used in other Windows development environment. The dynamic link library provides 44 layers of joint information of both hands and the identified gestures to the upper application layer.

Under the premise of collecting the data of 100 frames per second ,the time of single hand gesture being fixed in the screen is about 750 milliseconds in the case of gestures being static, which can be identified if the gesture jitter is not greater than $5 \mathrm{~cm}$; the time of both hands gesture being fixed in the screen is about 200 milliseconds in the case of gestures being static, and the distance between the hands is $10 \mathrm{~cm}$.

When the distance between one hand and the gesture recognition device is greater than $28 \mathrm{~cm}$, and the hand is in the palm state, the system recognizes the forward operation; when the distance between the one hand and the gesture recognition device is between 0 and $23 \mathrm{~cm}$, and the hand is in the palm state, the system recognizes the backward operation. And when the distance between the one hand and the gesture recognition device is $7 \mathrm{~cm}$, and the hand is in the palm state, the operation is recognized as leftward; when the distance between the one hand and the gesture recognition device is $7 \mathrm{~cm}$ from the center of the camera, Palm state, the system is identified for the right operation.

When the task of testing gesture recognition is successful, the gesture symbol will be displayed.And it won't be displayed if not. And the gesture recognition time is counted and the number of times of error recognition, as shown in the figure 3.

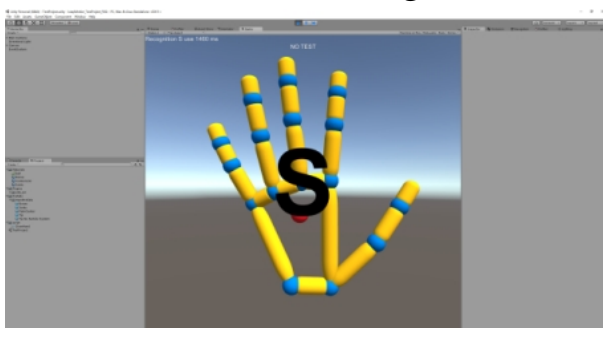

a) gesture symbol displayed if successful

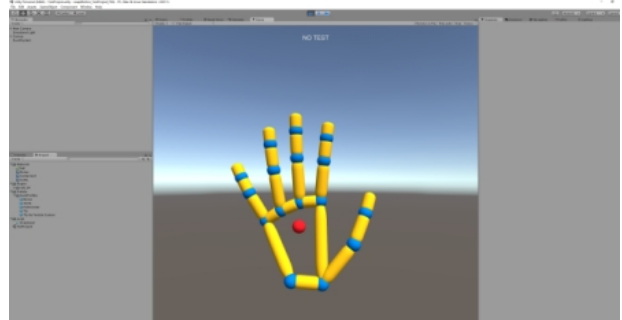

b) gesture symbol displayed if failed

Fig.3 Gesture recognition experiment performance 


\section{Experimental Design}

There is a significant question that how to compactly definite the achievement of a task which affects the calculation of time limitation, the number of mistakes, and effectiveness. The major points are the standards of successful task and the potential results in every task. If the task meets failure of participants or an unrecognized gesture, will be closed soon. When the participants asking help for the assistant, over the time limitation, multiple failures, and the impatient feeling from participants, the task will be stopped.

Before the start of the virtual reality helmet using, we display the VR equipments to the subjects , including the VR helmet, mobile phone, data cable, computer.Then we introduce the working principle of VR and the identification principle of the gesture recognition to them, and let them watch the virtual helmet introduction video to make sure the subjects are fully aware of virtual reality equipment. After that we open the prepared program, put the phone into the virtual reality helmet interface, and help the subjects wear the virtual reality helmet before their eyes. The focal length and the interpupillary distance is adjusted to a comfortable position using the button on the helmet, and the hands are put on the chest being the posture of pushing. Then we check if the position of the gesture recognizer is correct. After a series of adjustments, the users can begin to experience the virtual reality helmet, the using situation shown in Figure 5.
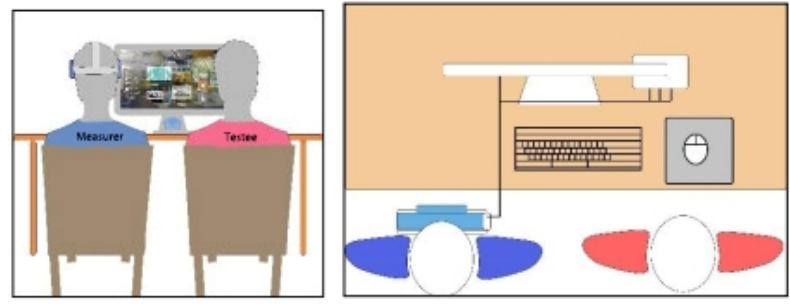

a) front view of use schematic

b) top view of use schematic

Fig.4 use schematic

The virtual reality helmet experiments are divided into two kinds, and one is the performance test, in which the main tester controls the operations of the subjects in the virtual scene through the computer. And the other is that subjects complete the use of function through the gesture interaction thus completing the user emotional test. In the first mode, in the case of the specified gesture, the subjects operates in the scene. The gesture symbols appear when the task is completed. To reduce the difficulty of using the virtual reality helmet, the basic operations are controlled by the main tester. And the second gesture mode of operation is to provide users the right of experiencing the function freely,in which the user completes the task in the scene through the gesture to complete the user emotional test.

The performance measurement experiment is to measure the performance availability of VR gesture recognition. The quantization value of learnability and usability is obtained in the evaluation system through the development of tasks and testing the completion of the task of the participants, the collect metrics of which is the task completion time, the number of errors and efficiency.

We plan to recruite 20 tested subjects in the experiment, aged between 20 and 30 years old. The 20 subjects are asked for completing the " gesture recognition in the roaming scene " experimental task in the specific scene. And the performance measurement experiment is made for each subject, recording the time of completing each task, the number of errors and efficiency. Finally, the user emotional test is completed and the score is obtained.

\section{Extracting of natural gestures}

Gesture refers to the way people communicate in their daily lives, and they share a small amount of information exchange as a complement for the communication between human and human. However, in some special occasions, gestures play a great role as a kind of signal language. The traffic gestures used by the traffic police are that they make different directions of different positions of static or dynamic gestures through the cooperation of the arms and hands, to give the drivers on the road the action information, so that road access can be orderly. 
In the gesture state of the device recognition, 10 sets of gestures in the roaming state are extracted, including the left and right hand gestures and the rotation function of one hand gestures in the roaming state, as shown in Table 2 , the gesture of the roaming scene.

Table 2 Gesture chematic description in a roaming scene

\begin{tabular}{|c|c|c|c|c|c|}
\hline Numbering & $\mathrm{A} 1$ & $\mathrm{~A} 2$ & $\mathrm{~A} 3$ & $\mathrm{~A} 4$ & A5 \\
\hline \multicolumn{6}{|l|}{ Gesture diagram } \\
\hline Gesture symbol & $\mathrm{W}$ & $\mathrm{S}$ & A & $\mathrm{D}$ & $\mathrm{W}$ \\
\hline Numbering & A6 & A7 & A8 & A9 & A10 \\
\hline \multicolumn{6}{|l|}{ Gesture diagram } \\
\hline Gesture symbol & $S$ & $\mathrm{~A}$ & $\mathrm{D}$ & I & $\mathrm{J}$ \\
\hline
\end{tabular}

\section{Data Analysis}

\section{Analysis of performance measurement experiment data}

After the performance measurement experiment is completed, the average task time, the number of errors and the efficiency index are calculated from the formulas (1-2) to (1-4), and the experimental data are obtained. Figure 6 shows the experimental data.

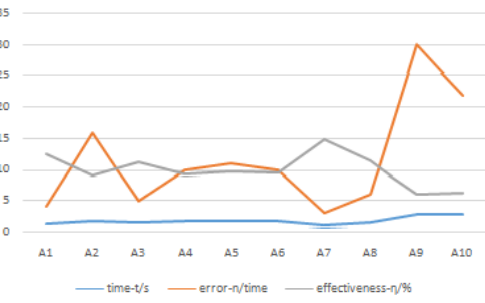

Fig.5 Average result line chart of Gesture recognition performance measure

As can be seen from the above figure, the number of errors for A9, A10, A2 is the largest, indicating that its usability is inferior to other gestures, and A1, A7, A3 and A8 have the least number of errors, indicating that the usability is better than others. According to the average time,A7,A1,A8,A3 have the shortest time,indicating its usability is good, and A9,A10, A2, A6 have the longest time, indicating that the user recognition is not good; A7, A1, A8, A3 have the highest efficiency, which is user-friendly, and A9, A10 have the lowest efficiency, which is not easy to use. The left and right gesture recognition is not very different through the analysis of the data of performance measurement gesture recognition, which forward, left, right to identify the best, and two hands to identify the rotation is the most poor, followed by backward operation.

\section{Analysis of user emotional test data}

In order to compare the users' emotional feeling when the perceived efficiency of different gestures recognition is different, the ACR5 scoring criteria are used to measure the psychological sensation of the 20 subjects tested during gestures using. Figure 7 shows the experimental process. 


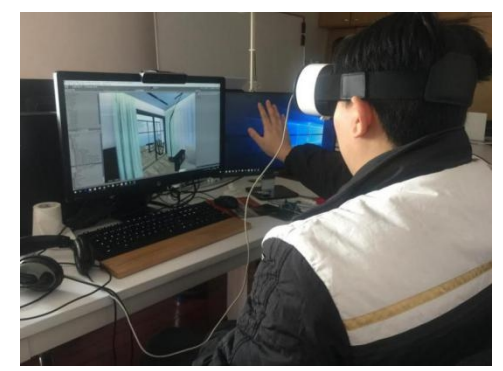

Fig.6 Diagram of experimental process

For the test of the identification efficiency, the subjects operate each gesture of one hand and two hands in the center of the recognition area. Each subject use the gesture in front of the reader in the right order, as shown in Table 2.Then we change the gesture and record the number and points, thus obtaining the data of each subject using the gesture and the feeling,as shown in Figure 8.

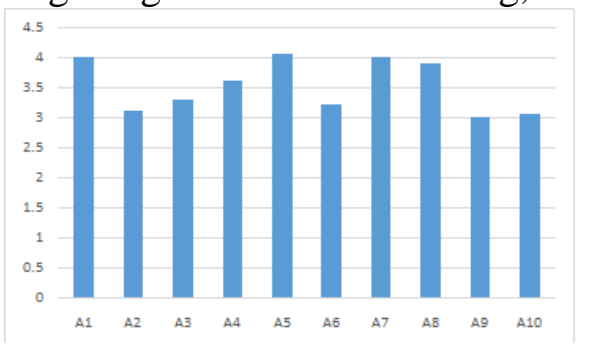

Fig.7 Average score of user emotional test

There are many influencing factors about the psychological feeling of people using the gestures, which is difficult to accurately define. In general it is related with that if the gesture itself is consistent with the rules of arm and finger movements, the recognition efficiency of the gestures in the reader and the gesture complexity,. The score is divided into five points to be measured to judge their own.The accurate figures are used to express the fuzzy user emotions, as a basis for consideration. From the histogram shown in Figure 8, the scores of A5, A1, A7, A8 gestures are the highest, and the A9 and A10 gestures account for the lowest. The A5-A8 score was relatively high. Users prefer simple gesture recognition, which can be analyzed, and the experience of two hands gestures operating is poor.

\section{Experimental results}

In this paper, through the experiment of the performance measurement, the analysis of user emotional test, and the quantifying of the indicators in the evaluation system using the method of performance measurement and user emotional test, the best simple gesture operation are obtained. We can find that the difference between left and right hand gesture recognition is not very big, which used hand has almost no affect on. Among them, the recognition and usability of forward, left, right operation is the best, while that of both hands to identify the rotation is poor, followed by backward operation.

In summary, this paper completes the construction of the interactive experience performance measurement system of the basic gesture recognition VR system, quantifies the user experience index, and outputs the quantification value of the comprehensive evaluation of the user experience. The subject needs to be improved and extended from the following ideas: (1) On the basis of the existing, we will further expand and improve the evaluation system and performance tasks according to the user habits and changes of the market trends.

(2) We will make the research method suitable for early research of the gesture development in the further development of the VR gestures recognition in the future. 


\section{Acknowledgement}

This work was financially supported by the National Natural Science Foundation of China (51305099).

\section{References:}

[1] Baker G.Distortion and risk in optimal in optimal incentive contracts 0.The Journal of Human Resources,2002,37(4):728-751.

[2]Ben Ammar Lassaad, Mahfoudhi Adel. Early usability evaluation in model driven framework[C]. 15th International Conference on Enterprise Information Systems, 2013:23-30.

[3]Lottridge, Danielle, Chignell, Mark. Emotional majority agreement: A psychometric property of affective self-report instruments[C]. TIC-STH'09: 2009 IEEE Toronto International Conference Science and Technology for Humanity. 2009(9):795-800. 